\title{
Existence and stability of solutions of the cubic complex Ginzburg-Landau equation with delayed Raman scattering
}

\author{
M. Facão* \\ Departamento de Física, Universidade de Aveiro and I3N, Campus Universitário de Santiago, 3810-193 Aveiro, Portugal \\ M. I. Carvalho ${ }^{\dagger}$ \\ DEEC/FEUP and INESCPorto, Universidade do Porto, Rua Dr. Roberto Frias, 4200-465 Porto, Portugal
}

(Received 14 June 2015; published 24 August 2015)

\begin{abstract}
We found two stationary solutions of the cubic complex Ginzburg-Landau equation (CGLE) with an additional term modeling the delayed Raman scattering. Both solutions propagate with nonzero velocity. The solution that has lower peak amplitude is the continuation of the chirped soliton of the cubic CGLE and is unstable in all the parameter space of existence. The other solution is stable for values of nonlinear gain below a certain threshold. The solutions were found using a shooting method to integrate the ordinary differential equation that results from the evolution equation through a change of variables, and their stability was studied using the Evans function method. Additional integration of the evolution equation revealed the basis of attraction of the stable solutions. Furthermore, we have investigated the existence and stability of the high amplitude branch of solutions in the presence of other higher order terms originating from complex Raman, self-steepening, and imaginary group velocity.
\end{abstract}

DOI: 10.1103/PhysRevE.92.022922

PACS number(s): 05.45.Yv, 42.65.Tg, 02.60.Lj

\section{INTRODUCTION}

The complex Ginzburg-Landau equation (CGLE) has been used to model several physical systems that are at the emergence of spatiotemporal patterns. Examples in optics are pulse propagation in fibers with linear and nonlinear gain and spectral filtering [1], pulse generation in fiber lasers with additive pulse mode-locking [2], or pulse propagation in hollow core photonic crystal fibers filled with resonant gases [3]. The CGLE is one of the known models that supports dissipative solitons and is usually presented in two forms, the cubic and quintic forms. Apart from the chirp-free or arbitrary amplitude solutions, which only exist in special curves of the parameter space, the cubic CGLE also admits fixed amplitude chirped solitons. However, these solutions are only stable on a certain region of the parameter space where the linear gain is positive. The linear gain being positive imposes background instability, which is a drawback for the observation of the stable solitons [4].

Generalizations of the CGLE have also been studied, some of those include a term that, in optics, models the delayed Raman scattering. For instance, the addition of this term has proved to stabilize the eruption solitons of the quintic CGLE [5-7]. Here we show that the cubic CGLE plus this same term admits two traveling solutions, one of them being stable, in certain parameter regions where the linear gain is negative. We investigate those solutions, namely, the parameter region of existence, amplitude and chirp profiles, velocity and stability. We further study the robustness of the stable solutions against other higher order perturbations such as self-steepening, imaginary group velocity, or imaginary Raman term. The latter higher-order terms are motivated by the study of pulse propagation at resonant frequencies of gases [3,8].

\footnotetext{
*mfacao@ua.pt

${ }^{\dagger}$ mines@fe.up.pt
}

\section{TRAVELING SOLUTIONS}

Consider the evolution equation,

$$
\begin{aligned}
i q_{Z}-\frac{D}{2} q_{T T}+s|q|^{2} q= & i \delta q+i \beta q_{T T}+i \epsilon|q|^{2} q+\xi q_{T} \\
& +R\left(|q|^{2}\right)_{T} q-i S\left(|q|^{2} q\right)_{T},
\end{aligned}
$$

where, in the optical context, $q$ is the normalized envelope of the optical field, and $Z$ and $T$ are the normalized propagation distance and retarded time, respectively. The parameters in this equation are all normalized versions of the actual parameters, namely, $\delta$ stands for linear gain or loss, $\beta$ for spectral filtering, $\epsilon$ for nonlinear gain, $\xi$ for the imaginary part of the group velocity, $R$ for the delayed Raman scattering, and $S$ for the selfsteepening effect, where $R$ and $S$ are allowed to be complex, i.e., $R=R_{r}+i R_{i}$ and $S=S_{r}+i S_{i}$. The parameters $D$ and $s$ may only take the values $\pm 1, D=1$ if the dispersion is normal and -1 if the dispersion is anomalous and $s=1$ or $s=-1$ for positive or negative Kerr effect, respectively.

We will restrict our analysis here to the case $D s=-1$, such that the left-hand side of Eq. (1) becomes the focusing nonlinear Schrödinger equation (NLS). We first applied the standard inverse scattering method perturbation analysis $[9,10]$ by considering the fundamental soliton of the NLS, namely, $q(T, Z)=$ $\eta(Z) \operatorname{sech}\left\{\eta(Z)\left[T-T_{0}(Z)\right]\right\} \exp [-i k(Z) T+i \zeta(Z)]$, and obtained the following equations for $\eta$ and $k$ :

$$
\begin{gathered}
\frac{d \eta}{d Z}=2 \eta \delta+\frac{4}{3} \epsilon \eta^{3}-\frac{2}{3} \beta \eta\left(\eta^{2}+3 k^{2}\right)-2 k \eta \xi-\frac{4}{3} \eta^{3} k S_{i}, \\
\frac{d k}{d Z}=-\frac{4}{3} \beta \eta^{2} k-\frac{2}{3} \eta^{2} \xi-\frac{8}{15} R_{r} \eta^{4}-\frac{4}{5} S_{i} \eta^{4} .
\end{gathered}
$$

The main results of this article concern pulse solutions of Eq. (1) with $\xi=0, R_{i}=0$, and $S=0$, such that it should be assumed that they have these values unless it is said explicitly. Under these conditions, and taking also $\beta>0$ and $\delta<0$, the latter to guarantee stable background, the equilibrium 


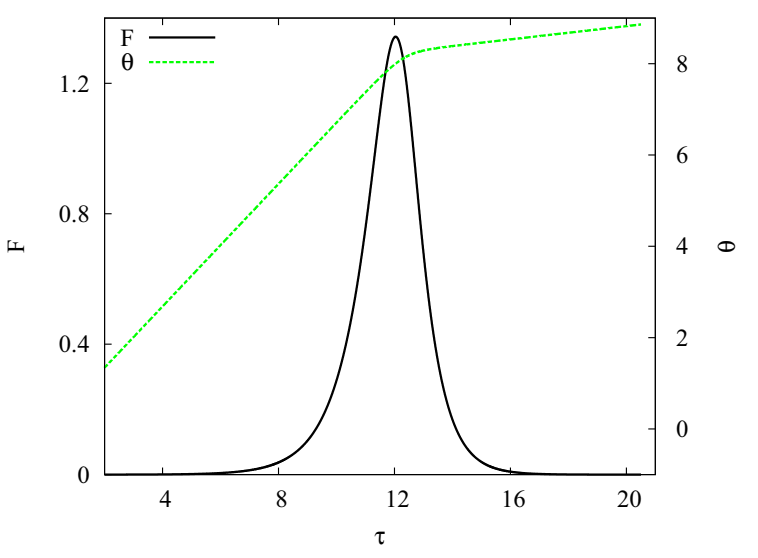

(a)

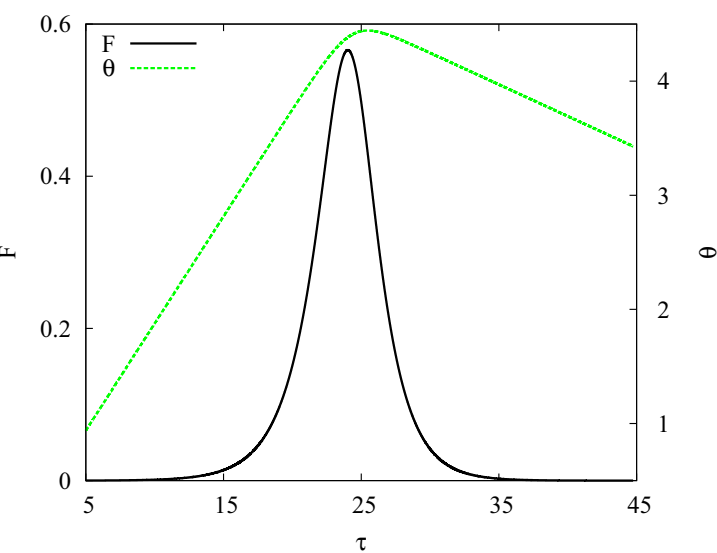

(b)

FIG. 1. (Color online) Amplitude profile and phase for $\delta=-0.012, \beta=0.3, \epsilon=0.2$, and $R_{r}=0.2$ (a) high-amplitude and (b) lowamplitude solutions.

nontrivial amplitude and frequency are given by

$$
-\frac{4 R_{r}^{2}}{25 \beta} \eta_{e}^{4}+\frac{1}{3}(2 \epsilon-\beta) \eta_{e}^{2}+\delta=0 \quad \text { and } \quad k_{e}=-\frac{2}{5 \beta} R_{r} \eta_{e}^{2},
$$

which yields two positive amplitudes if $2 \epsilon-\beta>$ $12 / 5\left|R_{r}\right|(|\delta| / \beta)^{1 / 2}$. Thus, according to this perturbation approach, as we introduce the Raman term the NLS fundamental soliton continues to exist but a new solution that has higher amplitude also appears. The above perturbation results are valid as long as $\delta, \beta, \epsilon$, and $R_{r}$ are small. However, we know that for $R=0$, the NLS soliton survive for parameters $\delta, \beta$, and $\epsilon$ that are not small, being the fixed amplitude chirped solution of the cubic CGLE [11]. The analytical form of these solutions was recently generalized for the case $\xi \neq 0$ [3]. They exist above a certain curve $\epsilon_{\lim }^{R=0} \equiv \epsilon(\beta)$ if $\delta+\xi^{2} / 4 \beta<0$ and below that curve otherwise. Unfortunately, they are only stable whenever the background is unstable. As we shall show below, if we introduce the Raman term this solution continues to be unstable. Hence, we are most interested on the other solution that direct numerical integration of Eq. (1) shows that, in some cases, is stable.

To further continue our search for solutions of Eq. (1), we write $q(Z, T)$ as a traveling solution of the form $q(Z, T)=$ $F(\tau) e^{i \theta(\tau)+i \omega Z}$, with $\tau=T-v Z$, where both $F$ and $\theta$ are real, in which case we obtain the following equations:

$$
\begin{aligned}
& \left(\frac{1}{2}+2 \beta^{2}\right) F^{\prime \prime}+\left[2 \beta v+D \xi+D\left(2 R_{r}+3 S_{i}\right) F^{2}\right. \\
& \left.\quad+2 \beta\left(2 R_{i}-3 S_{r}\right) F^{2}\right] F^{\prime}+(2 \beta \delta+D \omega) F \\
& \quad-(D v-2 \beta \xi) \frac{M}{F}-\left(\frac{1}{2}+2 \beta^{2}\right) \frac{M^{2}}{F^{3}}+(-D s+2 \beta \epsilon) F^{3} \\
& \quad+\left(D S_{r}+2 \beta S_{i}\right) F M=0, \\
& \left(\frac{1}{2}+2 \beta^{2}\right) M^{\prime}+\left[2 \beta v+D \xi+\left(D S_{i}-2 \beta S_{r}\right) F^{2}\right] M \\
& \quad+\left[D v-2 \beta \xi+D\left(2 R_{i}-3 S_{r}\right) F^{2}-2 \beta\left(2 R_{r}+3 S_{i}\right) F^{2}\right] \\
& \quad \times F F^{\prime}+(-2 \beta \omega+D \delta) F^{2}+(2 \beta s+D \epsilon) F^{4}=0,
\end{aligned}
$$

where $M=F^{2} \theta^{\prime}$. Since we are looking for localized solutions, $F, F^{\prime}$, and $M$ should tend to zero at the tails, where they obey the linearized version of Eq. (2). Since our numerical simulations indicate that the traveling solitons have constant phase derivative in their tails, we assume that $F(\tau)=F_{0} \mathrm{e}^{g \tau}$ and $M(\tau)=\mu F_{0}^{2} \mathrm{e}^{2 g \tau}$, with $g$ real and positive for $\tau \rightarrow-\infty$, and real and negative for $\tau \rightarrow+\infty$. Inserting these ansatz into the linearized version of Eq. (2) we obtain the following equations for $g$ and $\mu$ :

$$
\begin{gathered}
\left(\frac{1}{2}+2 \beta^{2}\right) g^{2}+(2 \beta v+D \xi) g-\left(\frac{1}{2}+2 \beta^{2}\right) \mu^{2} \\
-(D v-2 \beta \xi) \mu+2 \beta \delta+D \omega=0 \\
\mu=\frac{(2 \beta \xi-D v) g-D \delta+2 \beta \omega}{g+4 \beta^{2} g+2 \beta v+D \xi} .
\end{gathered}
$$

Note that $\mu$ is the value of $\theta^{\prime}$ at infinity and, since it depends on $g$, it is different at each tail. The pulse solutions that may exist in this system are in fact heteroclinic orbits in the phase space $\left(F, F^{\prime}, M\right)$.

We are interested in the parameter space where $\delta+$ $\xi^{2} / 4 \beta<0$, which, as it will be shown in next section, corresponds to stable background. To obtain the high amplitude solutions predicted by the perturbation approach and by direct numerical integration of Eq. (1), we use estimates for $\omega$ and $v$ from the numerical integration of Eq. (1) for a particular set of parameters for which the solution is stable and use a shooting method to find the profiles for the amplitude $F$ and phase derivative $\theta^{\prime}$. Our shooting method starts at the two tails and does the matching at the pulse peak location. The initial conditions at both ends were $F=F_{0} \mathrm{e}^{g_{1,2} \tau}=\epsilon \ll 1$, $F^{\prime}=g_{1,2} \epsilon$, and $M=\mu \epsilon^{2}$, where $g_{1,2}$ are the real solutions of Eq. (3). We have started with $\delta=-0.012, \beta=0.3$, $\epsilon=0.2$, and $R_{r}=0.2$ [see Fig. 1(a)] and find all the other solutions by continuation. We have studied the existence of the high-amplitude solutions for $R_{r} \neq 0$ on the plane $(\beta, \epsilon)$. The map of existence for $\delta=-0.012$ and $R_{r}=0.2$ on the region defined by $0<\beta<1$ and $0<\epsilon<0.4$ is shown in Fig. 2. There is a boundary of existence from below at which the high-amplitude solution merges with the low-amplitude solution and at which both cease to exist. The existence of this boundary is also predicted by the perturbation analysis performed on the NLS. In effect, this boundary corresponds to 


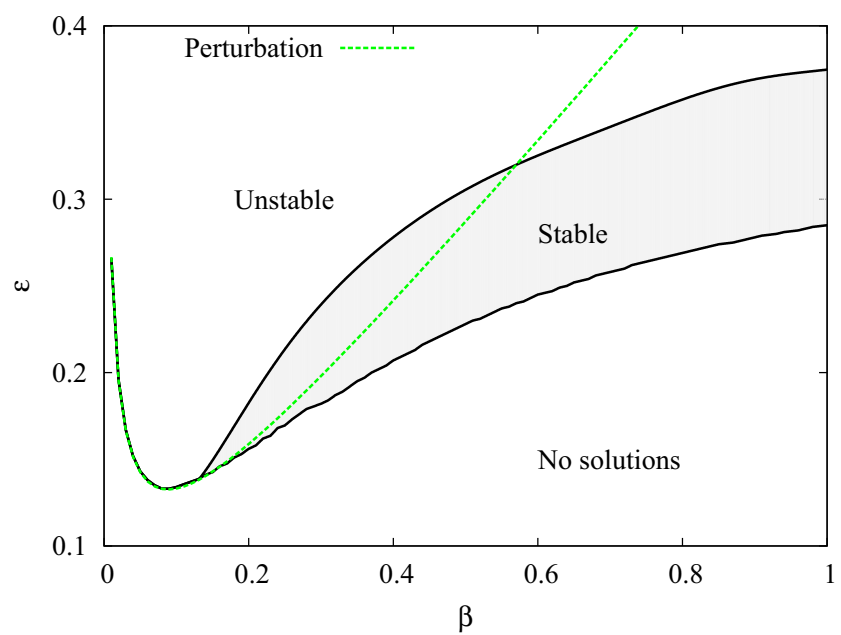

FIG. 2. (Color online) Existence and stability of the high amplitude solutions on the $(\beta, \epsilon)$ plane for $\delta=-0.012$ and $R_{r}=0.2$. The existence boundary from perturbation approach is shown as a dashed (green) curve.

the condition $2 \epsilon-\beta=12 / 5\left|R_{r}\right|(|\delta| / \beta)^{1 / 2}$, in which case we get two equal solutions for $\eta_{e}$. This curve is also represented in Fig. 2 and it coincides with our numerical existence curve for small $\beta$ but departs substantially from the latter for larger $\beta$. Then we have searched for solutions on the plane $\left(R_{r}, \epsilon\right)$. Figure 3 shows the region of existence on the region $0<R_{r}<0.65$ and $0<\epsilon<0.4$ for $\delta=-0.012$ and $\beta=0.3$, where we see the same kind of scenario as in Fig. 2. These results anticipate that in the space of parameters $\left(\beta, R_{r}, \epsilon\right)$ there is a minimum threshold $\epsilon_{\lim } \equiv \epsilon\left(\beta, R_{r}\right)$ for these solutions to exist. The same occurs for $R_{r}=0$ in which case $\epsilon_{\lim }^{R=0}=\beta\left(3 \sqrt{1+4 \beta^{2}}+D s\right) /\left(4+18 \beta^{2}\right)$ [3]. For $R_{r} \neq 0$ this limiting value of $\epsilon$ is larger but tends to the latter value as $R_{r}$ tends to zero.

We have used the same shooting procedure to obtain the low amplitude solutions but the first estimates for $v$ and $\omega$ cannot

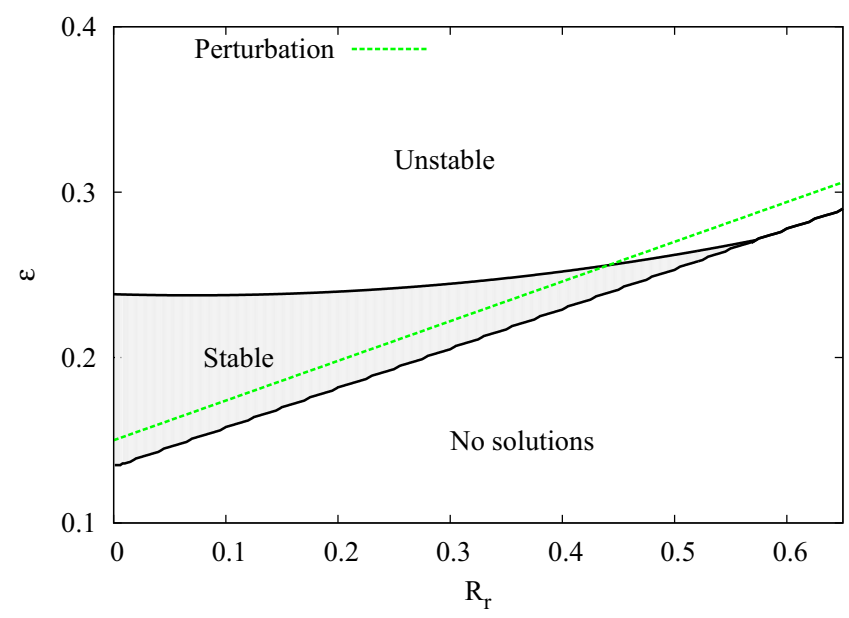

FIG. 3. (Color online) Existence and stability of the highamplitude solutions on the $\left(R_{r}, \epsilon\right)$ plane for $\delta=-0.012$ and $\beta=0.3$. The existence boundary from perturbation approach is shown as a dashed (green) curve.
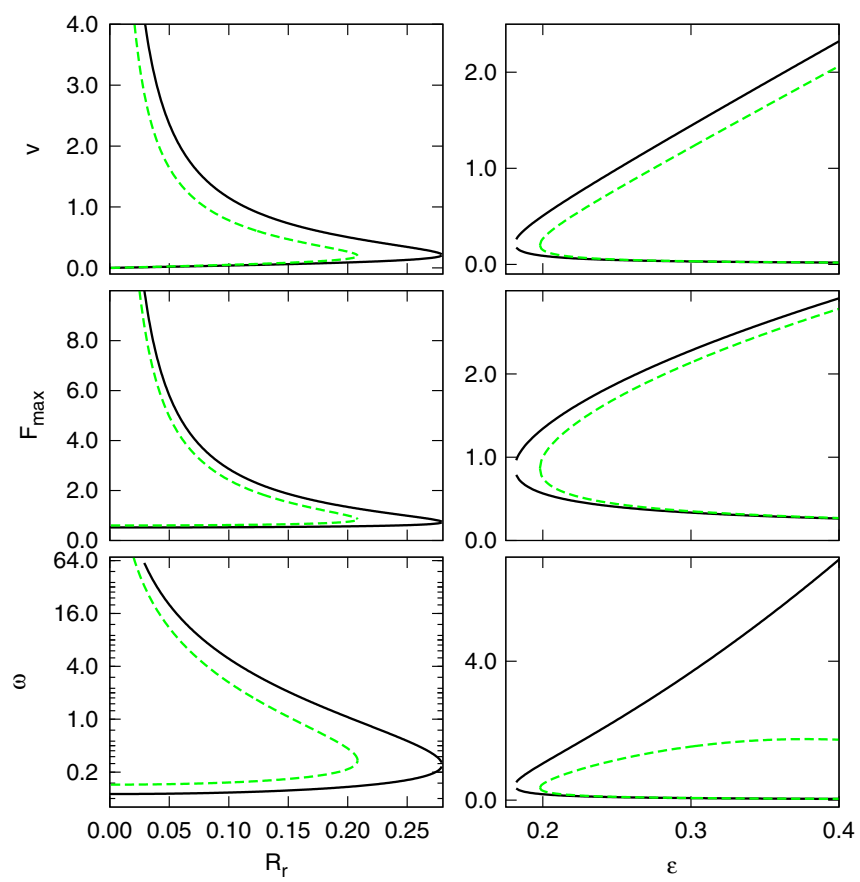

FIG. 4. (Color online) Velocity, peak amplitude, and propagation constant dependence on $R_{r}$ (left column), for $\delta=-0.012, \beta=0.3$, and $\epsilon=0.2$, and on $\epsilon$ (right column), for $\delta=-0.012, \beta=0.3$, and $R_{r}=0.2$. The high-amplitude solutions are the upper curves and the low-amplitude solutions are the lower curves. Solid lines (black) are results from shooting and dashed lines (green) are results from perturbation.

be obtained from the integration of Eq. (1), since the lowamplitude solutions are not stable. Hence, we have obtained the low-amplitude solutions by continuation from the lowexistence boundary or from the analytical solutions at $R=0$. One of those solutions is shown in Fig. 1(b).

We have studied the dependence of $v, \omega$, and peak amplitude for high- and low-amplitude solutions on the parameters $R_{r}$ and $\epsilon$. In the left column of Fig. 4 we show that $v, \omega$, and the peak amplitude $F_{\max }$ of the high-amplitude solitons decrease with the magnitude of $R_{r}$, whereas the opposite is true for the low-amplitude solitons. As represented, a similar behavior results from the perturbation analysis [for the equilibrium solution we have $v=-k_{e}$ and $\left.\omega=\frac{1}{2}\left(\eta_{e}^{2}-k_{e}^{2}\right)\right]$. For $R_{r}$ around 0.28 the solution reaches the boundary of existence referred to above, so that the high-amplitude solution equals the low-amplitude solution. Note that the point $R_{r}=0.28$ and $\epsilon=0.2$ is at the boundary of the region of existence in Fig. 3 ( $\beta=0.3$ ). However, a lower limiting $R_{r}$ value is obtained from perturbation results. In the right column of the same figure, we show results for the velocity, propagation constant, and peak amplitude for fixed $\delta, \beta$, and $R_{r}$ but varying $\epsilon$, also for both high- and low-amplitude solutions. A good agreement is found with the perturbation approach as far as the velocity and peak amplitude are concerned, but not in the propagation constant of the high-amplitude branch.

As referred in the last paragraph, the high-amplitude solutions have $F_{\max }, v$, and $\omega$ that grow as $R_{r}$ tends to zero. Since they do not exist at $R_{r}=0$ and our results show a rapid 


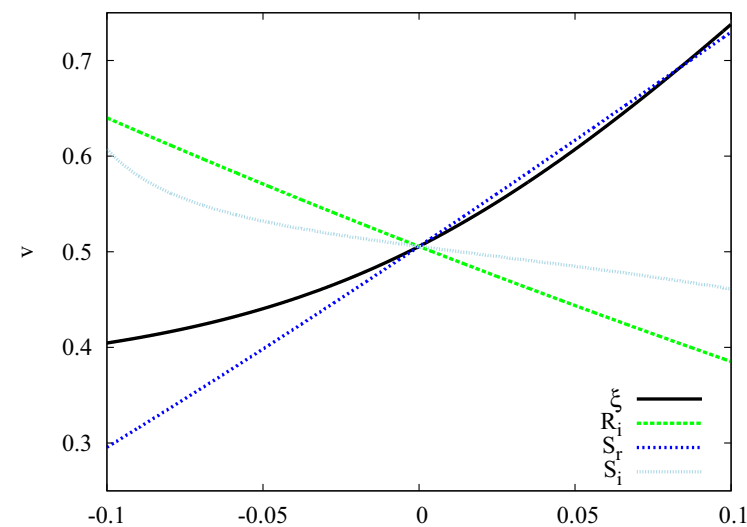

(a)

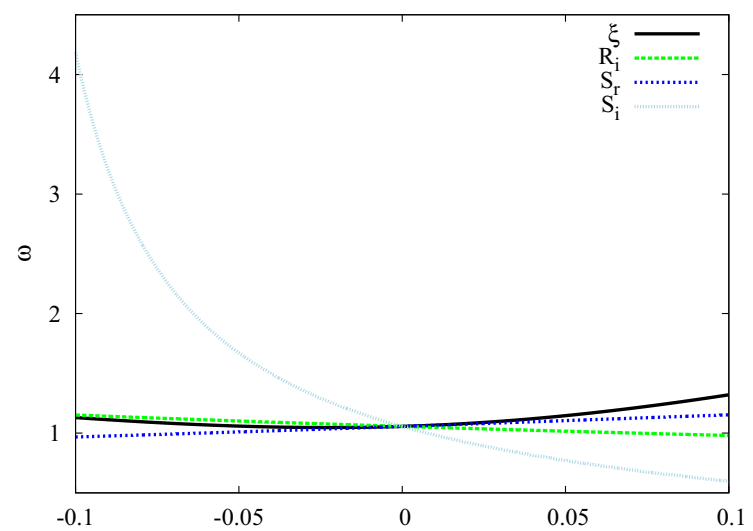

(b)

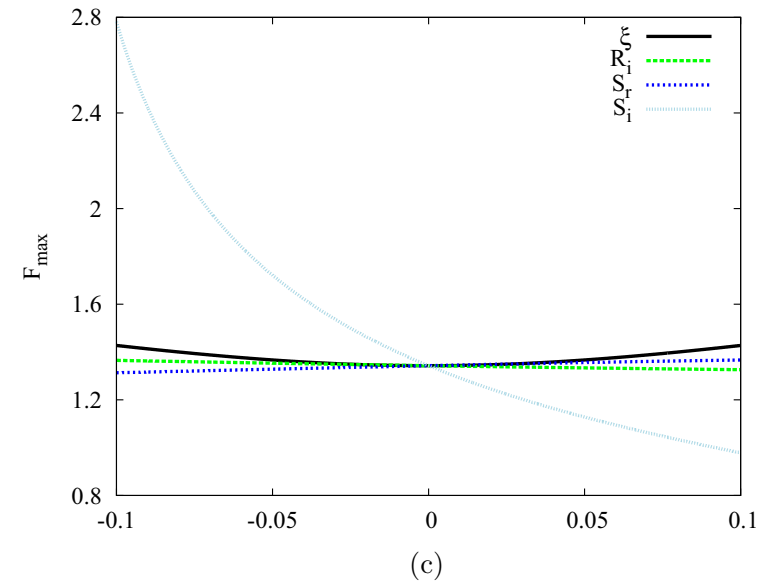

FIG. 5. (Color online) Characteristics of the high-amplitude solution for nonzero $\xi, R_{i}, S_{r}$, and $S_{i}$ and $\delta=-0.012, \beta=0.3, \epsilon=0.2$, and $R_{r}=0.2$. The $x$ axis represent each of the referred parameters as in the curve legends.

growth rate, especially for $\omega$, we suggest that they tend to infinity at this boundary. Nevertheless, our shooting results indicate that they tend to infinity at a slower pace for higher $\epsilon$ and more rapidly for $\epsilon$ close to $\epsilon_{\text {lim. }}$. At the latter case, the high values of these parameters are only obtained for very small $R_{r}$. As noted above, for $R_{r}=0$ the boundary of existence should coincide with $\epsilon_{\lim }^{R=0}$ and there the highamplitude solution merges with the low-amplitude solution, such that both should have infinity peak amplitude, velocity, and propagation constant. In fact, the peak amplitude and propagation constant of the cubic CGLE solutions tend to infinity as $\epsilon \rightarrow \epsilon_{\lim }^{R=0}$, but for $\xi=0, v$ is always zero.

We have also searched for solutions for nonzero $\xi, R_{i}, S_{r}$, and $S_{i}$. We have added each one separately and changed them from -0.1 to 0.1 , for the case $\delta=-0.012, \beta=0.3, \epsilon=0.2$, and $R_{r}=0.2$. The resulting velocity, propagation constant, and peak amplitude of the high-amplitude solutions are shown in Fig. 5. Despite doing all the calculations using $D=-1$ and $s=1$, we should note that if we keep $D s=-1$ but interchange $D$ and $s$, we will obtain equivalent solutions as long as we also change the sign of $R_{r}, S_{i}$, and $\xi$. By equivalent solutions, we mean the same amplitude profile and velocity and symmetric $\omega$ and $M$.

\section{SPECTRAL STABILITY}

We proceeded with the study of the solutions found in Sec. II by analyzing their linear stability spectrum. For this purpose, let us consider the above traveling solution plus a small perturbation term, $q(Z, T)=\left[F(\tau) e^{i \theta(\tau)}+\Delta(Z, \tau)\right] e^{i \omega Z}$. Demanding that $\Delta$ has exponential dependence on $Z$, i.e., $\Delta(Z, \tau)=u(\tau) e^{i \lambda Z}+x^{*}(\tau) e^{-i \lambda^{*} Z}$, we obtain the following stability eigenvalue problem:

$$
L \mathbf{w}=\lambda \mathbf{w}, \quad \mathbf{w}=\left(\begin{array}{ll}
u & x
\end{array}\right)^{T},
$$

where

$$
\begin{aligned}
L & =\left(\begin{array}{cc}
\left(-\frac{D}{2}-i \beta\right) \partial_{\tau \tau}-(i v+\xi) \partial_{\tau}-\omega-i \delta+A(\tau) & B(\tau) \\
-B^{*}(\tau) & \left(\frac{D}{2}-i \beta\right) \partial_{\tau \tau}-(i v-\xi) \partial_{\tau}+\omega-i \delta-A^{*}(\tau)
\end{array}\right), \\
A(\tau) & =2(s-i \epsilon) F^{2}-R\left(3 F F^{\prime}+F^{2} \partial_{\tau}-i \theta^{\prime} F^{2}\right)+2 i S\left(2 F F^{\prime}+F^{2} \partial_{\tau}\right), \\
B(\tau) & =\left[(s-i \epsilon) F^{2}-R\left(F F^{\prime}+F^{2} \partial_{\tau}+i \theta^{\prime} F^{2}\right)+S\left(2 i F F^{\prime}-2 \theta^{\prime} F^{2}+i F^{2} \partial_{\tau}\right)\right] \mathrm{e}^{2 i \theta} .
\end{aligned}
$$




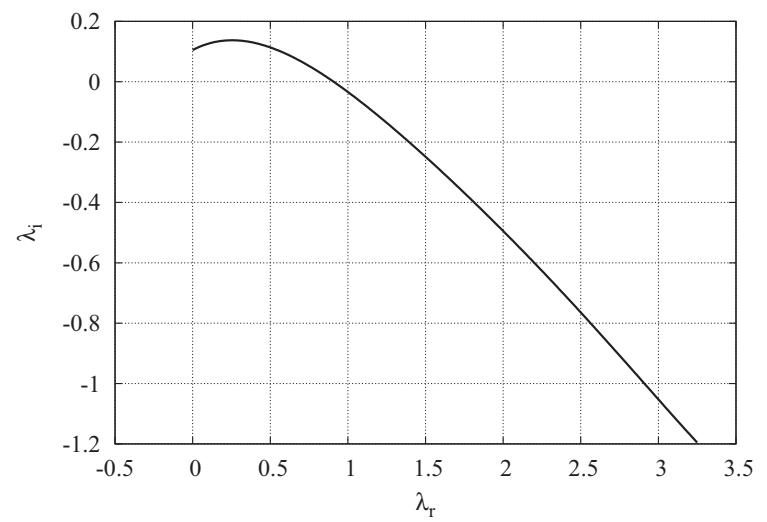

(a)

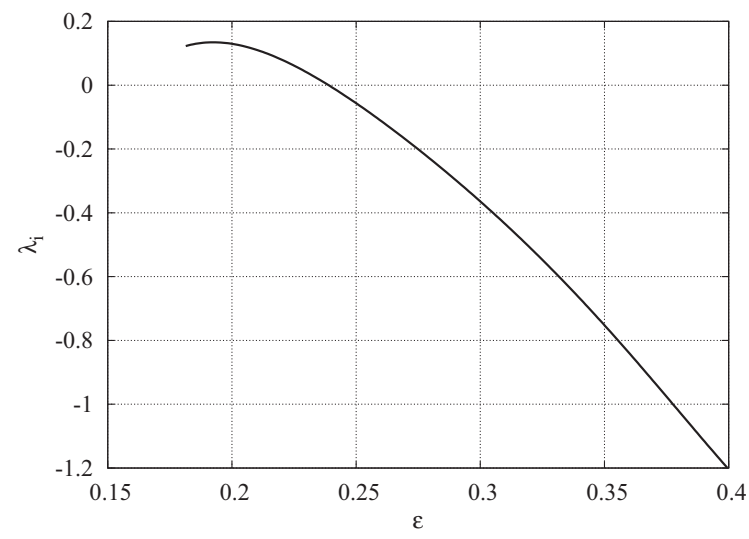

(b)

FIG. 6. (a) Trajectory of the discrete eigenvalue (the one with positive real part) as $\epsilon$ increases. (b) Imaginary part of the same eigenvalue as function of $\epsilon$. Both graphs correspond to high-amplitude solutions and $\delta=-0.012, \beta=0.3$, and $R_{r}=0.2$.

The traveling pulses are linearly stable if $L$ has no eigenvalues $\lambda$ with negative imaginary part. Due to the symmetry of Eq. (5) relative to the imaginary axis, we expect that whenever there

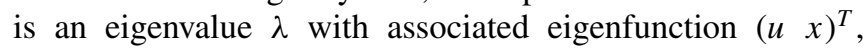
there also exists an eigenvalue $-\lambda^{*}$, corresponding to the eigenfunction $\left(x^{*} u^{*}\right)^{T}$. Therefore, in the following, only the eigenvalues with zero or positive real part will be considered. Moreover, the spectrum of $L$ consists of a continuous set and discrete eigenvalues. One trivial discrete eigenvalue is $\lambda=0$, which is double and corresponds to the translational and rotational invariances of Eq. (1). The location of the continuous spectrum may be estimated using the continuous spectrum of the limiting form of $L$ for $\tau \rightarrow \pm \infty$, which is obtained from $L$ putting $A(\tau)=B(\tau)=0$. The continuous spectrum of $L_{\infty}$ is composed of two oblique parabolas on the $\lambda$ plane defined by $(D / 2+i \beta) r^{2}+(v-i \xi) r-\omega-i \delta=\lambda$ and $(-D / 2+i \beta) r^{2}+(v+i \xi) r+\omega-i \delta=\lambda$, with $r$ being any real number. These parabolas are in the upper half-plane for $\delta+\xi^{2} / 4 \beta<0$. Following Henry [12], the continuous spectrum of $L$ itself is on the regions defined by the lines that constitute the continuous spectrum of $L_{\infty}$, so that it is on or inside the above parabolas. Hence, the continuous stability spectrum of these solutions is stable if $\delta+\xi^{2} / 4 \beta<0$, as we stated in the previous section.

We searched for discrete eigenvalues using the approach of Alexander et al. [13] of the Evans function method [14]. Due to the analyticity of the Evans function away from the continuous spectrum, the existence of unstable eigenvalues was investigated by calculating the Evans function around a semicircle with large radius whose straight line stands on the real axis and then applying the argument principle. In order to avoid the $\lambda=0$ eigenvalue, we have deformed the semicircle close to the origin using another semicircle, this one with a very small radius. To confirm our results, we have also computed these eigenvalues by considering the discretized operator $L$, which was obtained by evaluating Eq. (5) in equidistant points and using finite-differences and then applying sparse matrix methods.

The regions of stability of the high-amplitude solutions in the parameter space $\left(\beta, R_{r}, \epsilon\right)$ and $\delta=-0.012$ were evaluated in the planes $R_{r}=0.2$ and $\beta=0.3$ as shown in Figs. 2 and 3 .
In both figures, the stable regions are for smaller $\epsilon$, close to the existence boundary. Figure 2 shows that they are stable above a certain threshold of $\beta$, on the other hand, Fig. 3 shows that these solutions are stable from $R_{r}=0$ up to a certain threshold of $R_{r}$. Following the trajectory of the discrete eigenvalues, we have found that as $\epsilon$ is increased, up to the stability boundary, a pair of eigenvalues cross the real axis and enter the unstable half-plane. On the other hand, as $\epsilon$ decreases toward the boundary of existence, these two complex eigenvalues merge at the imaginary axis (see Fig. 6), giving rise to two pure imaginary eigenvalues that split; one stays at the positive part of the imaginary axis, so it is stable, the other one travels in the direction of the negative part of the axis passing through zero. At this $\epsilon$, the high-amplitude solutions touch the low-amplitude solutions. The low-amplitude solutions are unstable for all the parameters in the studied region. They have two discrete eigenvalues, apart from the zero one, both are pure imaginary but one is stable and the other one is unstable. It is worthwhile to compare these results with the ones obtained for the cubic-quintic CGLE regarding different branches and change of stability [15]. In fact, as in Ref. [15] our results show two branches of solutions for the same parameters and that the local edges of soliton existence in the $\epsilon$ or $R_{r}$ parameters correspond to change of stability. Nevertheless, there is also change of stability away from local edges of existence, namely, inside the high-amplitude branch.

Let us give a rough physical explanation for the above results. In the case of $R_{r}=0$, there are solutions for $\epsilon>0$ above the curve $\epsilon_{\lim }^{R=0}$ for $\delta<0$ that reveal a balance of the nonlinear gain with the linear loss and filtering. However, those solutions are unstable. The introduction of the Raman term brings another solution that is higher in peak amplitude, shorter in duration, and has higher chirp, such that it suffers stronger filtering since it is steeper in amplitude and phase. This stronger filtering stabilizes this new solution, at least up to a certain threshold nonlinear gain, i.e., up to the threshold of stability that was found here. The same reasoning may explain why the high-amplitude solution have higher peak amplitude and shorter duration for higher $\epsilon$, in order to increase the filtering associated loss and thus balance the nonlinear gain. 


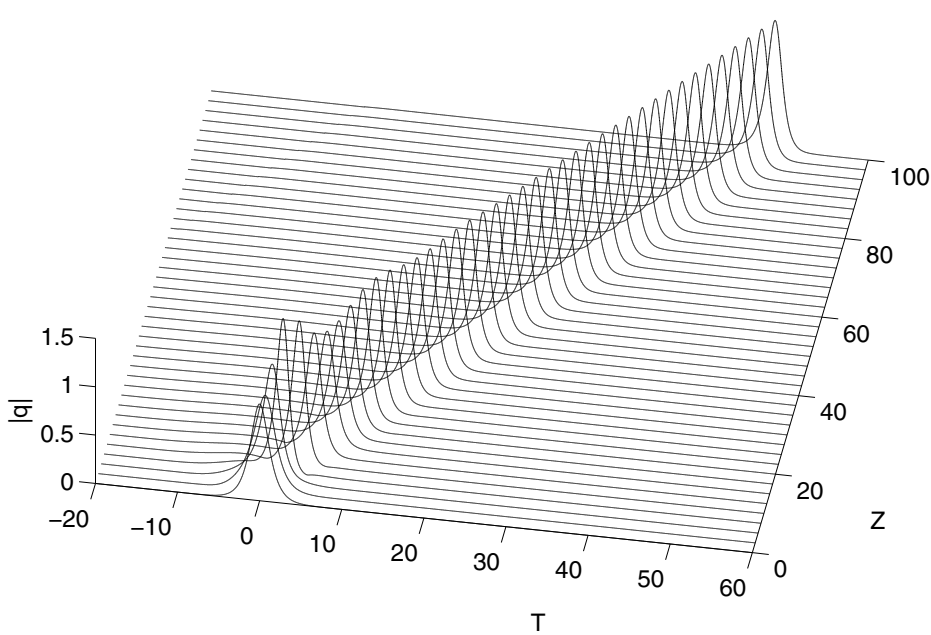

(a)

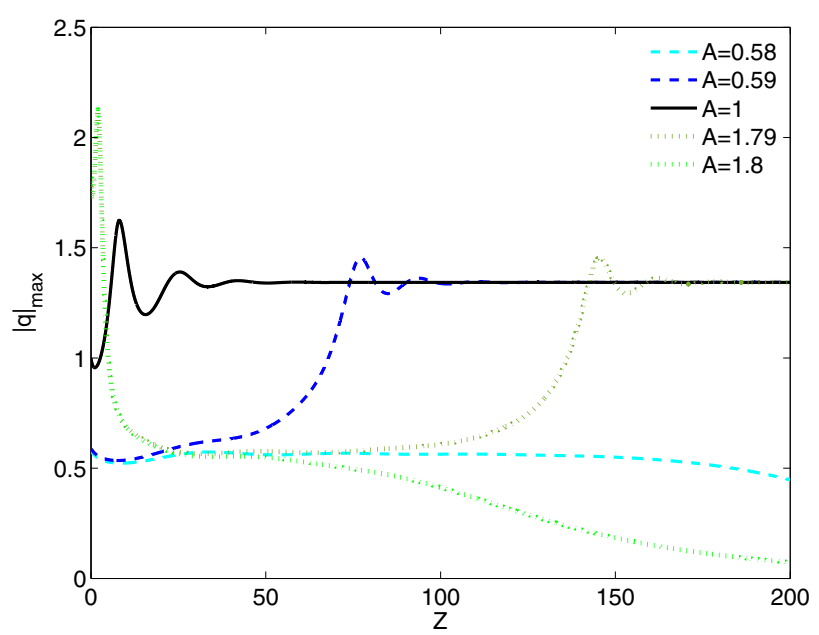

(b)

FIG. 7. (Color online) Propagation for $\delta=-0.012, \beta=0.3, \epsilon=0.2$, and $R_{r}=0.2$ (a) $\operatorname{sech}(T$ ) at the input (b) peak amplitude for $A \operatorname{sech}(A T)$ at the input.

\section{NUMERICAL SIMULATIONS}

We have investigated pulse evolution in the three regions represented in Figs. 2 and 3, by numerically solving Eq. (1) using $\operatorname{sech}(T)$ at the input in order to better excite any existing unstable mode. In all cases, we have considered $\delta=-0.12$, $\beta=0.3$, and $R_{r}=0.2$. As a first example, we let $\epsilon=0.2$, which is well within the stable region for this set of parameters. As Fig. 7(a) shows, after some initial adjustments in both amplitude and width, the pulse profile evolves rapidly toward the high-amplitude solution, propagating steadily from that point on without appreciable changes in both beam profile and trajectory. Actually, our numerical simulations have shown that, within the stable region, this convergence to the stable solution can be achieved for inputs of the type $A \operatorname{sech}(A T)$, with $A$ typically ranging from the amplitude of the lowamplitude solution to a maximum value that can be several times the peak amplitude of the stable soliton. This maximum

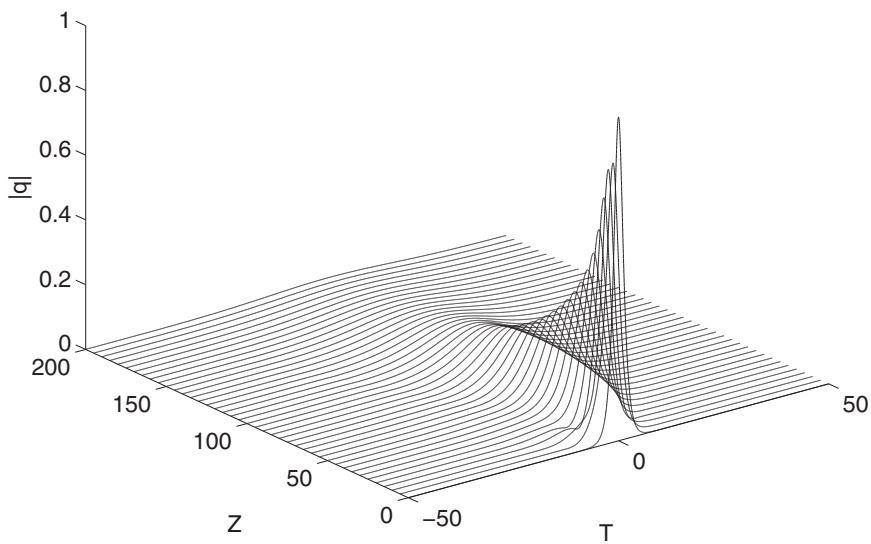

(a) value depends on the localization within the stable region, being larger close to the lower boundary of the existence region. Figure 7(b) shows the peak-amplitude evolution for the previous parameters and for different values of $A$, indicating that in this case the stable solution is able to attract sech profiles with amplitudes between approximately 0.59 and 1.79. Note that, for the set of parameters considered, the high- and low-amplitude solutions have peak-amplitude values of 1.34 and 0.57 , respectively.

As expected, in the other two regions, the unstable one and the one where no solutions are allowed, the $\operatorname{sech}(T)$ is not be able to reach a steady propagation. Figure 8(a) depicts the evolution for $\epsilon=0.15$ (no solution region). In this case, the pulse broadens and the peak amplitude decreases down to complete disappearance of the pulse. A different behavior is represented in Fig. 8(b) for $\epsilon=0.28$ (unstable region). In this case, the initial stages of evolution are characterized by a fast increase of peak amplitude, which can be explained

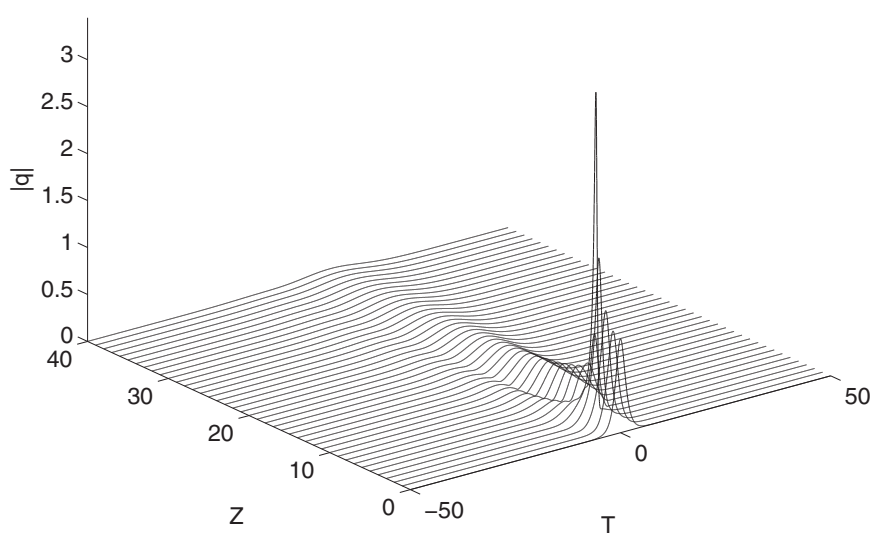

(b)

FIG. 8. Propagation for $\delta=-0.012, \beta=0.3$, and $R_{r}=0.2$ with $\operatorname{sech}(T)$ at the inputs (a) $\epsilon=0.15$ (no solution region) and (b) $\epsilon=0.28$ (unstable region). 
by the growth of unstable modes. After this initial burst, the subsequent evolution is analogous to the one for $\epsilon=0.15$, with the pulse peak amplitude decreasing at approximately the same rate in both cases.

We have also simulated pulse propagation using Eq. (1) for $\xi, R_{i}, S_{r}$, and $S_{i}$ different from zero. In order to have a preliminary idea of the stability of the solutions that were found in Sec. II (Fig. 5), we have tested the cases $\xi= \pm 0.1, R_{i}= \pm 0.1, S_{r}= \pm 0.1$, and $S_{i}= \pm 0.1$ also for $\delta=-0.012, \beta=0.3, \epsilon=0.2$, and $R_{r}=0.2$. In all the cases, we have obtained stable propagation. Moreover, in all except the $S_{i}=0.1$ case, the input $\operatorname{sech}(T)$ evolved to the high-amplitude solution obtained with the shooting. For the $S_{i}=0.1$ case, we needed to add a phase to the sech input in agreement with the velocity retrieved from the shooting results in order to observe stable propagation. Nevertheless, a detailed investigation of the stability of the solutions for nonzero $\xi, R_{i}$, $S_{r}$, and $S_{i}$ is postponed for a future work using parameter ranges associated with a practical application of this model.

\section{v. CONCLUSIONS}

Motivated by physical systems described by generalizations of the cubic CGLE, we searched for stable soliton solutions of one of these generalizations, namely, one that includes a delayed Raman scattering term. In the anomalous dispersion regime and positive Kerr effect, or vice versa, a perturbation approach around the soliton of the NLS equation shows that, in a certain region of the parameter space, there are two solutions. In fact, we have obtained the ordinary differential equation for the traveling solutions of the generalized cubic CGLE and found these two solutions using a shooting procedure. Then, we studied the spectrum of their linear stability operator using the Evans function approach and performed direct numerical simulations of the evolution equation to confirm the previous results and to obtain basis of attraction of the stable solutions. One branch of solutions, the ones with lower peak amplitude, is the continuation of the CGLE chirped solutions and continues unstable. The other branch consists of solutions of higher peak amplitude and they are stable from the existence boundary up to a threshold of nonlinear gain. The linear stability spectrum has one unstable eigenvalue in the case of the low-amplitude solutions and a pair of unstable eigenvalues in the case of the high-amplitude solutions above the stability threshold. A preliminary study of existence and stability of pulse solutions of a cubic CGLE with Raman and other higher-order terms was made, yielding stability in all the studied cases. A careful analysis of that case as well as the case of anomalous dispersion and negative Kerr effect will be considered in a future work.

\section{ACKNOWLEDGMENTS}

This work was partially supported by Fundação para a Ciência e a Tecnologia through Projects No. UID/CTM/50025/2013 and UID/EEA/50014/2013.
[1] M. Matsumoto, H. Ikeda, T. Uda, and A. Hasegawa, J. Lightwave Technol. 13, 658 (1995).

[2] H. Haus, J. Fujimoto, and E. Ippen, J. Opt. Soc. Am. B 8, 2068 (1991).

[3] M. Facão, S. Rodrigues, and M. I. Carvalho, Phys. Rev. A 91, 013828 (2015).

[4] N. Akhmediev and A. Ankiewicz, Dissipative Solitons, Lecture Notes in Physics (Springer, Berlin, 2005).

[5] S. Latas and M. Ferreira, Opt. Lett. 35, 1771 (2010).

[6] M. Facão, M. I. Carvalho, S. C. Latas, and M. F. Ferreira, Phys. Lett. A 374, 4844 (2010).

[7] M. Facão and M. Carvalho, Phys. Lett. A 375, 2327 (2011).

[8] G. Huang, L. Deng, and M. G. Payne, Phys. Rev. E 72, 016617 (2005).
[9] D. J. Kaup and A. C. Newell, Proc. R. Soc. London A 361, 413 (1978).

[10] V. I. Karpman, Phys. Scr. 20, 462 (1979).

[11] N. N. Akhmediev, V. V. Afanasjev, and J. M. Soto-Crespo, Phys. Rev. E 53, 1190 (1996).

[12] D. Henry, Geometric Theory of Semilinear Parabolic Equations, 3rd ed., Lecture Notes in Mathematics, Vol. 840 (SpringerVerlag, Berlin, 1993).

[13] J. Alexander, R. Gardner, and C. Jones, J. Reine Angew. Math. 410, 167 (1990).

[14] M. Facão and M. I. Carvalho, Theoret. Math. Phys. 160, 917 (2009).

[15] J. Soto-Crespo, N. Akhmediev, and G. Town, Opt. Commun. 199, 283 (2001). 